\title{
Is there an independent effect of polycystic ovary syndrome (PCOS) and menopause on the prevalence of subclinical atherosclerosis in middle aged women?
}

\author{
Evelyn O Talbott \\ Jeanne Zborowski \\ Judy Rager \\ Juley R Stragand \\ Department of Epidemiology, \\ Graduate School of Public Health, \\ University of Pittsburgh, Pittsburgh, \\ PA, USA
}

Correspondence: Evelyn O Talbott University of Pittsburgh, Department of Epidemiology, I 30 DeSoto St./A526 Crabtree Hall, Pittsburgh,

PA I526I, USA

Tel + | 4I26243074

Fax +| 4126247397

Email eotl@pitt.edu

\begin{abstract}
Polycystic ovary syndrome (PCOS), a common reproductive endocrine condition manifests at puberty, and is characterized by hyperandrogenism, chronic anovulation, and obesity. PCOS cases exhibit an adverse coronary heart disease (CHD) profile at an early age, including insulin resistance, dyslipidemia and increased central adiposity. It can be hypothesized that the menopausal transition, whether natural or surgical, may provide an additional "insult", resulting in greater cumulative risk to their vasculature. Coronary artery calcification (CAC), a measure of subclinical atherosclerosis (SCA), was measured by electron beam tomography in 149 PCOS cases and 166 controls (mean age 47.3 and 49.4 respectively). Cases had a higher prevalence of CAC (63.1\%) compared to controls $(41.0 \%)$, $(\mathrm{p}=0.037)$ after adjustment for age and BMI. A total of 22 cases and 39 controls had undergone natural menopause, 12 cases and 26 controls underwent surgical menopause (with biochemical confirmation) and 115 cases and 101 controls reported being currently premenopausal. There was a significant difference in CAC values between cases and controls in all three-menopause categories including pre-menopausal, surgically induced and natural menopause $(\mathrm{p}<0.001)$. Duration since menopause (years) and use of hormone replacement therapy were not different between cases and controls for the two menopause groups. Logistic regression was carried out with CAC ( $\leq 10$ vs $>10)$ as the dependent variable, and independent variables: PCOS status, current age, BMI, and menopausal status, (pre-menopause, surgical and natural menopause) and selected CHD risk factors. The data indicate that women with PCOS exhibit significantly increased CAC compared to controls after adjustment for age and BMI and menopausal status. PCOS status and fasting glucose were significant risk factors for CAC $(\mathrm{p}<0.05)$. Both natural and surgical menopause were independent risk factors for CAC as well $(\mathrm{p}<0.01)$. HDLT was of borderline significance, $\mathrm{p}<0.10$. Further follow-up of this cohort will be valuable in determining whether PCOS status continues to affect cardiovascular risk as they undergo the menopausal transition.
\end{abstract}

Keywords: polycystic ovary syndrome, coronary artery calcification, subclinical atherosclerosis, coronary vasculature, coronary heart disease risk, menopausal transition

\section{Introduction}

PCOS is the most common reproductive endocrine condition affecting $6 \%-10 \%$ of all women (Knochenhauer et al 1998; Azziz et al 2004). It is a lifelong condition, which manifests at puberty and is characterized by obesity, hyperandrogenism, chronic anovulation and menstrual irregularity. Women with PCOS are hyperinsulinemic and fully $40 \%$ of cases will develop type 2 diabetes by age 50 (Dunaif 1995; Ehrman et al 1999). Two recent independent investigations in both younger (25-34) (Christian et al 2003) and older (35-62) (Talbott et al 2004) women with PCOS demonstrated an increased prevalence of coronary artery calcium (CAC) measured by electron beam 
tomography. These increases were found to be independent of age and body mass index. These findings reflect structural changes in the coronary vasculature. However other researchers (Lakhani et al 2004; Orio et al 2004; Vural et al 2004; Meyer et al 2005) have also noted significant functional changes in young PCOS cases compared to controls reflected in reduced endothelial function and pulse wave velocity. The results of these studies may reflect an early metabolic process, or "insult" beginning much earlier than in normal menstruating women.

There is a well-documented loss of the coronary protection noted in numerous studies of middle-aged women as they enter the menopausal transition (Sutton-Tyrrell et al 1998; Matthews et al 1989). At issue is whether this further insult promulgated on PCOS women results in a cumulative or additive effect on the prevalence of subclinical atherosclerosis. This may be reflected in increased central adiposity, increases in LDL, reduced HDLt and increased prevalence of hypertension. No study to date has considered the effect of menopause on women with PCOS.

Within the backdrop of this recent information on lifelong metabolic problems of younger PCOS women as they approach middle age, it can be hypothesized that as PCOS cases reach the menopausal transition, a second metabolic "insult" may be associated with a greater cumulative risk to their vasculature and increased subclinical atherosclerosis (SCA). Given the increase in CAC noted in these two smaller independent studies, the present study will consider the prevalence of CAC using EBT in a large group of well-characterized women with PCOS 35-60 years of age $(\mathrm{N}=149)$ and normal controls $(\mathrm{N}=166)$.

We hypothesize that women with PCOS cases compared to controls will (1) exhibit a greater increased prevalence of CAC independent of age and BMI, and further (2) that the menopausal transition will be associated with an increased prevalence of CAC in PCOS cases compared to controls. PCOS is a common condition and one that can potentially account for a significant amount of premature coronary heart disease in middle-aged women.

\section{Methods}

\section{Study population}

Details on the identification and recruitment of the original Pittsburgh PCOS cohort have been reported elsewhere (Talbott et al 1995). Briefly, women with PCOS were identified largely from two sources of records: (1) the practice records of physicians in the 1970-1993, and (2) through recruitment efforts aimed at private practices and the local chapter of the
PCOSA support group with physician confirmation. A clinical diagnosis of PCOS was established at baseline if there was (1) a history of chronic anovulation in association with either (2) evidence of clinical (hirsutism) or biochemical (total testosterone level greater than $2.0 \mathrm{nmol} / \mathrm{L}$ ) hyperandrogenism, or (3) a luteinizing hormone: follicle stimulating hormone ratio (LH:FSH) greater than 2.0. Originally, age- ( \pm 5 years) and race-matched neighborhood control subjects were selected by using a combination of voter's registration tapes from the Greater Pittsburgh area and Cole's Cross Reference Directory of Households (Cole's Cross Reference Directory 1993). The control population has continued to be of similar age and socioeconomic and ethnic status. In the present study, women with PCOS and controls who were 35-60 years of age were eligible for recruitment and invited to undergo non-invasive EBT of the coronary arteries (2003-2004). Also, any subject reporting a history of Type 1 (insulin-dependent) diabetes was excluded. A total of 149 cases and 166 controls were included in the baseline EBT and screening.

\section{Risk factor assessment}

Detailed data collection and laboratory methodologies have been published previously (Talbott et al 1995). Briefly, height was measured to the nearest half inch on a wall mounted Harpenden stadiometer; weight was measured to the nearest half pound. BMI, a measure of relative obesity, was calculated as a mathematical function of weight and height $\left(\mathrm{kg} / \mathrm{m}^{2}\right)$. Waist and hip circumferences (in $\mathrm{cm}$ ) were measured in duplicate with an inelastic tape at the level of the umbilicus and greater trochanter, respectively; the average value was recorded. The waist: hip ratio was calculated as the waist circumference divided by the hip circumference. Blood pressure was assessed in duplicate after a 30-minute caffeine restriction and 5-minute rest using a random-zero sphygmomanometer. A questionnaire was administered that included the evaluation of medical, surgical, menstrual and reproductive histories, current medication use, lifestyle factors, and family history of PCOS.

A 12-hour fasting blood sample was obtained for lipid and hormone assays. Serum concentrations of total cholesterol, HDLT, $\mathrm{HDL}_{2}$, and triglycerides were measured in the Heinz Lipid Laboratory at the University of Pittsburgh. Total cholesterol was determined by the enzymatic method of Allain et al (1974). HDLT was determined by the method above after selective precipitation by heparin/manganese and the removal by centrifugation of VLDL and LDLT. LDLc was calculated using the Friedewald formula (Friedewald et al 1972). Triglycerides were determined using the enzymatic 
procedure of Bucolo and David (1973). Plasma glucose was analyzed by using enzymatic assay (Yellow Springs Glucose Analyzer, Yellow Springs Instruments) and plasma insulin by radioimmunoassay. Type 2 diabetes history was obtained and was defined as either a doctor diagnosis of Type 2 diabetes or a fasting blood glucose of $>125 \mathrm{mg} / \mathrm{dl}$. History of use of cholesterol lowering medications and blood pressure medication was also obtained.

Natural menopausal status was defined as not having a period for 12 months or more when there was no medical cause other than menopause with or without hormone replacement therapy. Those who reported a hysterectomy with bilateral salpingo oophorectomy (BSO) were classified as surgical menopausal. Women, who reported removal of the uterus with one or two ovaries intact were classified as surgical if they reported a history of physician prescribed hormone replacement or had a biochemical profile of the reproductive hormones indicative of menopause was evaluated. This consisted of a FSH level greater that $25 \mathrm{mIU} / \mathrm{ml}$, and a progesterone level below $3 \mathrm{ng} / \mathrm{ml}$. This model was adapted from the algorithm applied in the WISE study (Women's Ischemia Syndrome Evaluation) (Johnson and Merz 2004). Individuals who did not meet these criteria were considered premenopausal.

\section{Coronary calcium assessment}

Coronary calcium was assessed at the University of Pittsburgh Medical Center (UPMC) Preventive Heart Care Center by EBT using the Imatron C-150 Ultrafast CT Scanner (Imatron, South San Francisco, CA, USA). For the coronary arteries, 30 to 40 contiguous $3 \mathrm{~mm}$-thick transverse images were obtained from the level of the aortic root to the apex of the heart during a maximal breath hold using ECG triggering so that each 100 millisecond exposure is acquired during the same phase of the cardiac cycle ( $60 \%$ of R-R interval). EBT technicians were blinded to the case/control status of the subjects. Only one scanning sequence was performed in order to minimize radiation exposure to the study participants.

Coronary calcium scores were generated using a Base Value Region of Interest (BVROI) computer software program (AcuImage). All pixels greater than 130 Hounsfield units and $1 \mathrm{~mm}^{2}$ within an operator defined region of interest in each $3 \mathrm{~mm}$ thick image within the coronary arteries were considered calcified. The Agatston method was used to calculate a calcium score for each region of interest by multiplying the area of all significant pixels by a grade number $(1,2,3$, or 4$)$ indicative of the peak CT number (ie, Hounsfield units). Coronary Artery Calcium volume scores were also generated.

\section{Statistical analyses}

All statistical analyses were performed using SPSS (version 13.0). Risk factors were obtained at the same time that the EBT was conducted during this 2001-2003 clinical assessment. Demographic, anthropometric, lipid and hormonal data were available for analysis from the baseline visit. Descriptive statistics, including measures of central tendency and dispersion, were computed for all variables of interest for PCOS cases and controls and compared using the nonparametric Mann-Whitney U test for continuous data or a chi-square or Fisher's exact test for categorical data. The distribution of coronary artery calcium was markedly skewed and could not be normalized using traditional mathematical transformations. The prevalence of coronary artery calcium was therefore modeled as a binary dichotomous variable.

Univariate logistic regressions was used to identify the baseline cardiovascular risk factors, including age, BMI, smoking, and hormone use, that potentially predicted CAC as the dependent binary variable ( $\leq 10$ vs $>10$ ). Logistic regression was conducted to explore the relationship between prevalent $\mathrm{CAC}$ as the dependent variables and age, BMI and PCOS status as independent variables assessed concurrently. Variables related to CAC in univariate analysis were included in the subsequent models. In the multivariable regression modeling, the effect of PCOS on CAC was assessed after adjustment for age and BMI.

\section{Results}

\section{Baseline and coronary heart disease risk factors}

A total of 321 women agreed to participate in this phase of the study (2000-2003). Results are reported on 315 women who met our previously stated age criteria. Participant characteristics (smoking status, hormone use, anthropometric measures, blood pressure, lipids, and insulin) at the time of baseline EBT (2000-2002) are presented by race in Table 1 for 149 PCOS cases and 166 controls. Fifty African American (AA) women (16 PCOS cases and 34 controls) were included in this assessment. Women with PCOS were slightly younger than control women: $47.3 \pm 5.6$ (mean \pm SD) years for PCOS cases and $49.4 \pm 5.8$ years for control women. There were no significant differences between PCOS cases and controls in the prevalence of current smoking, current use of hormones or oral contraceptives. PCOS women had higher mean BMI ( $32.6 \mathrm{vs} 28.3 \mathrm{~kg} / \mathrm{m}^{2}$ ), and larger waist circumference and waist/hip ratio than control women. Average systolic and diastolic blood pressures were similar for the two groups. 
Table I Descriptive statistics at baseline EBT (2000-2003) by race and PCOS status

\begin{tabular}{|c|c|c|c|c|c|c|}
\hline & \multicolumn{2}{|l|}{ All races ${ }^{a}$} & \multicolumn{2}{|l|}{ White } & \multicolumn{2}{|c|}{ African American } \\
\hline & $\begin{array}{l}\text { Cases } \\
n=149\end{array}$ & $\begin{array}{l}\text { Controls } \\
n=166\end{array}$ & $\begin{array}{l}\text { Cases } \\
(n=\mid 27)\end{array}$ & $\begin{array}{l}\text { Controls } \\
(n=132)\end{array}$ & $\begin{array}{l}\text { Cases } \\
(n=16)\end{array}$ & $\begin{array}{l}\text { Controls } \\
(n=34)\end{array}$ \\
\hline & $(\bar{x} / \mathbf{S D})$ & $(\bar{x} / \mathbf{S D})$ & $(\bar{x} / \mathrm{SD})$ & $(\bar{x} / \mathbf{S D})$ & $(\bar{x} / \mathbf{S D})$ & $(\bar{x} / \mathbf{S D})$ \\
\hline Age, years & $47.3^{\mathrm{b}}(5.6)$ & $49.4(5.8)$ & $47.5^{\mathrm{b}}(5.5)$ & $49.6(5.7)$ & $46.7(6.2)$ & $49.0(6.3)$ \\
\hline BMI, kg/m² & $32.6^{\mathrm{b}}(8.8)$ & $28.3(6.1)$ & $32.0^{\mathrm{b}}(8.4)$ & $27.3(5.4)$ & $37.8^{\mathrm{b}}(9.8)$ & $32.2(7.1)$ \\
\hline Waist/hip ratio & $0.84^{b}(0.09)$ & $0.80(0.08)$ & $0.84^{b}(0.09)$ & $0.79(0.08)$ & $0.86(0.08)$ & $0.85(0.07)$ \\
\hline Systolic BP, mm Hg & II $8.5(\mid 1.2)$ & II $6.9(14.3)$ & $117.9^{b}(11.2)$ & II5.3 (I3.4) & $123.2(\mid 1.9)$ & $123.2(16.0)$ \\
\hline Diastolic BP, mm Hg & $76.2(8.3)$ & $75.3(8.4)$ & $76.0(8.3)$ & $74.2(8.1)$ & $77.1(9.0)$ & $79.6(8.3)$ \\
\hline LDLc, mg/dl & $123.8(38.8)$ & $127.4(32.5)$ & I22.I (34.3) & $128.2(3 \mid .8)$ & $146.3(68.4)$ & $124.0(35.6)$ \\
\hline HDLc, mg/dl & $52.1^{\mathrm{b}}(15.0)$ & $57.2(14.2)$ & $52.3^{\mathrm{b}}(15.1)$ & $58.0(14.5)$ & $49.0(15.4)$ & $54.2(12.5)$ \\
\hline Triglycerides, mg/dl & $126.0^{\mathrm{b}}$ & 102.0 & $128.0^{\mathrm{b}}$ & 107.5 & 92.0 & 90.0 \\
\hline (median/range) & $(36.0,1563.0)$ & $(36.0,479.0)$ & $(36.8,1563.0)$ & $(45.0,479.0)$ & $(36.0,628.0)$ & $36.0,234.0)$ \\
\hline Fasting glucose, mg/dl & $98.3(22.6)$ & $93.3(11.5)$ & $97.5(21.1)$ & $92.3(9.6)$ & $99.9(17.8)$ & $97.4(16.6)$ \\
\hline Insulin, $\mu \mathrm{U} / \mathrm{ml}$ & $19.5^{b}(12.2)$ & $13.4(8.1)$ & $19.0^{b}(11.8)$ & $12.8(7.8)$ & $22.2^{\mathrm{b}}(11.9)$ & $15.9(9.1)$ \\
\hline \multirow[t]{2}{*}{ Duration yrs HRT use } & $3.7(3.9)$ & $5.6(5.3)$ & $3.85(3.5)$ & $5.26(4.5)$ & - & $7.87(8.3)$ \\
\hline & $(\mathrm{N} / \%)$ & $(\mathrm{N} / \%)$ & $(\mathrm{N} / \%)$ & $(\mathrm{N} / \%)$ & $(\mathrm{N} / \%)$ & (N/\%) \\
\hline Current smoker & $25(16.8)$ & $24(14.5)$ & $2 I(16.5)$ & $18(13.6)$ & $4(25.0)$ & $6(17.6)$ \\
\hline Current HRT use & $18(12.1)$ & $31(18.8)$ & $14(\mid 1.0)$ & $23(17.6)$ & $2(12.5)$ & $8(23.5)$ \\
\hline \multicolumn{7}{|l|}{ Menopause status } \\
\hline Premenopausal & II 5 (77.2) & $101(60.8)$ & $96(75.6)$ & $82(62.1)$ & $14(87.5)$ & $19(55.9)$ \\
\hline Natural menopause & $22(14.8)$ & $39(23.5)$ & $20(15.7)$ & $32(24.2)$ & $2(|2| .5)$ & $7(20.6)$ \\
\hline Surgical menopause & $12(8.1)$ & $26(15.7)$ & II (8.7) & $18(13.6)$ & $0(0.0)$ & $8(23.5)$ \\
\hline Type 2 diabetes & $18(12.1)^{b}$ & $3(1.8)$ & $15(11.8)^{b}$ & $\mathrm{I}(0.8)$ & $2(12.5)$ & $2(5.9)$ \\
\hline Hypertension, treated & $40(26.8)$ & $30(18.1)$ & $33(26.0)^{b}$ & $16(12.1)$ & $5(31.3)$ & $14(4 \mid .2)$ \\
\hline Lipid lowering meds & $13(8.7)$ & $7(4.2)$ & $13(10.2)$ & $6(4.5)$ & $0(0.0)$ & I (2.9) \\
\hline
\end{tabular}

Values are Mean/Count (SD/\%).

alncludes 5 women of other race and I woman missing race (all PCOS cases).

${ }^{\mathrm{b}} \mathrm{p}<0.05$ cases vs. controls. Test of significance is Mann Whitney for continuous variables; Chi-Square or Fisher Exact for categorical variables.

LDL cholesterol was similar for cases and control women; however, HDLc cholesterol was lower among PCOS cases and triglyceride levels were higher $(\mathrm{p}<0.05)$. Mean fasting glucose was not significantly different. However, PCOS women had higher mean insulin levels (19.5 vs $13.4 \mu \mathrm{U} / \mathrm{ml})$ $(\mathrm{p}<0.05)$. More controls than cases $(39.2 \%$ vs $22.9 \%)$ reported being currently postmenopausal, most likely a reflection of the older age of the controls.

PCOS cases were more likely to report a diagnosis of Type 2 diabetes $(p<0.05)$ and use of hypertensive and lipid lowering meds (n.s.). White women with PCOS had a mean age \pm SD of $47.5 \pm 5.5$ compared to $49.6 \pm 5.7$ years for white control women. Cases and controls were again similar with regard to current smoking, current use of hormones or oral contraceptives. White PCOS cases also had a significantly higher BMI than white controls (mean $32.0 \mathrm{v} 27.3 \mathrm{~kg} / \mathrm{m}^{2}$ ) and a larger waist circumference $(94.1 \mathrm{vs} 82.9 \mathrm{~cm})$ and waist/hip ratio (0.84 vs 0.79$)$ than controls. White PCOS women had higher systolic blood pressure compared to controls $(\mathrm{p}<0.05)$. Total cholesterol (not shown) and LDL cholesterol were similar for case and control women; however, HDLc was lower and triglycerides were higher among white PCOS cases compared to white controls. Moreover, PCOS cases had higher mean insulin levels (19.0 vs $12.8 \mu \mathrm{U} / \mathrm{ml})(\mathrm{p}<0.05)$. African American (AA) cases were younger than AA controls 46.7 years \pm 6.2 (mean $\pm \mathrm{SD}$ ) years compared to $49.0 \pm 6.2$ years for control women (n.s.). No significant differences were noted between AA cases and controls in current smoking, current use of hormones or oral contraceptives. African American PCOS women had higher BMI ( $\left.37.8 \mathrm{vs} 32.2 \mathrm{~kg} / \mathrm{m}^{2}\right)$ and larger waist circumference $(103.2$ vs $95.0 \mathrm{~cm} ; \mathrm{p}=$ n.s.) than their AA control counterparts; however, waist/hip ratio and both systolic and diastolic blood pressure showed no significant differences between the two groups.

A total of 22 cases and 39 controls had undergone natural menopause (no period within 12 months and no surgical intervention), 12 cases and 26 controls underwent surgical menopause (with biochemical confirmation of menopause) and 115 cases and 101 controls reported being premenopausal. Mean duration of hormone replacement use among cases was 3.7 years (3.9) and among controls 5.6 years (5.3) n.s. 


\section{Prevalence of coronary artery calcium in PCOS and controls}

The prevalence and distribution of CAC is shown for the total group and by race in Table 2 . The overall prevalence of CAC was $63.1 \%(94 / 149)$ among cases and $41 \%(68 / 166)$ among controls $(\mathrm{p}<0.05)$. The prevalence among white PCOS cases was $60.6 \%$ versus $35.6 \%$ in race-matched controls and 93.8\% among African American cases compared to $61.8 \%$ among AA controls $(\mathrm{p}<0.05)$. Moreover, a CAC score of greater than 10 was found in $35.5 \%$ of cases compared to $12.2 \%$ of controls ( $\mathrm{p}<0.05$ ). The prevalence of CAC Coronary Calcium scores ranged between 1.0 and 845.5 for 94 PCOS cases and between 1.0 and 211.1 for 86 controls among all subjects with prevalent CAC. Mean and median CAC scores were 26.8 and 3.1 respectively for PCOS cases and 6.3 and 0.0 respectively for controls $(\mathrm{p}<0.05)$. There was a statistically significant difference in CAC values between cases and controls in all three-menopause categories: pre-menopausal, surgically induced and natural menopause $(\mathrm{p}<0.001)$. The median CAC values were 1.42 and 0.0 for premenopausal, 14.7 compared to 0.0 for natural and 24.7 compared to 1.9 in surgical menopausal cases and controls respectively.

\section{Hormonal and reproductive characteristics of PCOS cases and controls and the distribution of coronary artery calcification}

Shown in Table 3 are the menopausal characteristics of PCOS cases and controls who met the criteria for either a natural or surgical menopause. The reported age of natural menopause was similar in cases compared to controls with the median 50.0 and 48.0 years respectively (n.s.). Duration of menopause for those who had undergone a natural menopause was 6.7 years vs. 6.3 years (n.s.) among controls. PCOS cases reported a younger age at surgical menopause compared to controls (42.5 vs 45.0); similarly they demonstrated higher CAC scores.

Duration in years since surgical menopause was 10.2 for cases and 11.7 for controls. Coronary artery calcium scores were significantly greater between cases and controls for both menopausal categories even though the age at baseline interview was two years older for cases (52.5 vs 54.2). Most notable were the elevated FSH levels and very low progesterone levels indicative of ovarian failure and menopausal status.

Shown in Figures 1-3 are the distributions of median CAC scores for cases and controls stratified by age and by menopause status. It can be noted that within the age strata $<45$, Figure 1 (premenopausal shown only) cases do not exhibit significantly greater median CAC compared to controls. In both the 45-54 categories and 55+ age strata (Figures 2 and 3), menopausal cases contrasted to their control counterparts exhibit increased median coronary calcium scores. Both natural and surgical PCOS cases demonstrate increased median CAC scores in the 45-54 age category ( 4.8 vs 0.0 for natural and 16.5 vs. 1.4 for controls) n.s. In the oldest age strata, 55+, cases have higher median CAC scores than controls in the two menopause categories (32.3 compared to 0.00 and 51.8 compared to 2.6 , respectively, $\mathrm{p}<0.05$ ).

Table 2 Prevalence and distribution of coronary artery calcium score by PCOS status and race

\begin{tabular}{|c|c|c|c|c|c|c|}
\hline & \multicolumn{2}{|l|}{ All races ${ }^{a}$} & \multicolumn{2}{|l|}{ White } & \multicolumn{2}{|c|}{ African American } \\
\hline & $\begin{array}{l}\text { PCOS } \\
(N=149)\end{array}$ & $\begin{array}{l}\text { Controls } \\
(\mathrm{N}=166)\end{array}$ & $\begin{array}{l}\text { PCOS } \\
(N=127)\end{array}$ & $\begin{array}{l}\text { Controls } \\
(N=132)\end{array}$ & $\begin{array}{l}\text { PCOS } \\
(N=16)\end{array}$ & $\begin{array}{l}\text { Controls } \\
(\mathrm{N}=34)\end{array}$ \\
\hline \multicolumn{7}{|c|}{$\begin{array}{l}\text { Distribution of CAC Agatson Scores } \\
(\mathrm{N} / \%)\end{array}$} \\
\hline 0 & $55(36.9)$ & $98(59.0)$ & $50(39.4)$ & $85(64.4)$ & I (6.3) & $13(38.2)$ \\
\hline I-9.9 & $41(27.5)$ & $45(27.1)$ & $38(29.9)$ & $34(25.8)$ & $3(18.8)$ & II (32.4) \\
\hline $10-49$ & $35(23.5)$ & $19(11.4)$ & $24(18.9)$ & $9(6.8)$ & $9(56.3)$ & $10(29.4)$ \\
\hline $50+$ & $18(12.1)$ & $4(2.4)$ & $15(11.8)$ & $4(3.0)$ & $3(18.8)$ & $0(0.0)$ \\
\hline Prevalence (any CAC) (N/\%) & $94(63.1)^{b}$ & $68(41.0)$ & $77(60.6)^{b}$ & $47(35.6)$ & I5 $(93.8)^{b}$ & $2 I(6 I .8)$ \\
\hline Prevalence $(\mathrm{CAC}>10)(\mathrm{N} / \%)$ & $54(35.5)$ & $20(12.2)$ & $37(29.0)$ & II (8.5) & $12(75.0)$ & $9(26.5)$ \\
\hline Mean & 26.8 & 6.3 & 26.2 & 6.2 & 39.0 & 6.8 \\
\hline Median & $3.1^{c}$ & 0.0 & $1.7^{c}$ & 0.0 & $15.6^{c}$ & 3.6 \\
\hline Range & $(I-845)$ & $(I-2 \mid I . I)$ & & & & \\
\hline
\end{tabular}

ancludes 5 women of other race and I woman missing race.

${ }^{b}$ Chi square $p<0.05$ for cases compared to controls.

cM-W test $p<0.05$ for cases compared to controls. 
Table 3 Characteristics of post-menopausal PCOS cases and controls

\begin{tabular}{|c|c|c|c|c|c|c|c|}
\hline & & \multicolumn{3}{|l|}{ PCOS cases } & \multicolumn{3}{|l|}{ Controls } \\
\hline & & $\begin{array}{l}\text { Natural } \\
\text { Menopause } \\
\mathbf{N}=\mathbf{2 2}\end{array}$ & $\begin{array}{l}\text { Surgical } \\
\text { Menopause } \\
N=12\end{array}$ & $\begin{array}{l}\text { Total } \\
\mathbf{N}=\mathbf{3 4}\end{array}$ & $\begin{array}{l}\text { Natural } \\
\text { Menopause } \\
\mathbf{N}=39\end{array}$ & $\begin{array}{l}\text { Surgical } \\
\text { Menopause } \\
N=\mathbf{2 6}\end{array}$ & $\begin{array}{l}\text { Total } \\
\mathbf{N}=65\end{array}$ \\
\hline Age at EBT & Mean/SD & $53.8(5.2)$ & $50.2(4.4)$ & $52.5(5.2)$ & $54.0(3.5)$ & $54.4(5.0)$ & $54.2(4.2)$ \\
\hline (years) & Median & 54.3 & 49.6 & 52.8 & 54.1 & 54.7 & 54.2 \\
\hline Age period & Mean/SD & $47.0(8.6)$ & $40.0(8.6)$ & $44.4(9.1)$ & $47.7(4.9)$ & $42.8(7.0)$ & $45.8(6.2)$ \\
\hline $\begin{array}{l}\text { stopped } \\
\text { (years) }\end{array}$ & Median & 50.0 & 42.5 & 44.5 & 48.0 & 45.0 & 47.0 \\
\hline Time since & Mean/SD & $6.7(6.5)$ & $10.2(9.9)$ & $8.0(7.9)$ & $6.3(4.5)$ & II.7 (7.8) & $8.4(6.5)$ \\
\hline $\begin{array}{l}\text { menopause } \\
\text { (years) }\end{array}$ & Median & 4.9 & 7.3 & 5.4 & 5.2 & 10.4 & 6.8 \\
\hline $\begin{array}{l}\text { CAC score } \\
\text { (Agatson) }\end{array}$ & Median & $16.3 *$ & $24.7 * *$ & $18.4^{*}$ & 0.0 & 1.9 & 0.0 \\
\hline $\begin{array}{l}\text { Duration of } \\
\text { hormone use } \\
\text { (years) }\end{array}$ & Median & $4.0 \mathrm{yrs}$ & $2.5 \mathrm{yrs}$ & & $5.0 \mathrm{yrs}$ & $5.0 \mathrm{yrs}$ & \\
\hline $\begin{array}{l}\text { FSH mlU/ml } \\
\text { (not currently } \\
\text { on HRT) }\end{array}$ & Median & 38.7 & 38.3 & 37.6 & 84.0 & 50.3 & 78.2 \\
\hline $\begin{array}{l}\text { Progesterone } \\
\mathrm{ng} / \mathrm{dl} \text { (no HRT) }\end{array}$ & Median & 1.4 & 2.65 & 1.5 & 1.2 & 1.65 & 1.3 \\
\hline $\begin{array}{l}\text { Estradiol } \\
\mathrm{pg} / \mathrm{ml} \text { (no HRT) }\end{array}$ & Median & 39.3 & 33.1 & 37.3 & 28.7 & 21.3 & 27.8 \\
\hline $\begin{array}{l}\text { Total } \\
\text { Testosterone } \\
\text { ng/dl (no HRT) }\end{array}$ & Mean (SD) & I.I (0.68) & $0.93(3.6)$ & I.I (0.63) & $0.75(47)$ & $0.72(0.09)$ & $0.7(4 I)$ \\
\hline
\end{tabular}

Logistic regression analysis: The logistic regression was conducted using a higher threshold of CAC score $\leq 10$ versus $>10$ in a dichotomous fashion. It was believed that this would be more appropriate for consideration of clinical significance. The final models are shown in Tables 4, 5, and 6 considering age, BMI, PCOS status as well as the menopausal categories: pre-menopausal (reference), natural, and surgical. Logistic model for cases and controls combined (Table 4) demonstrated a significant contribution of the association with coronary artery calcification of $\leq 10$ versus $>10$ CAC score

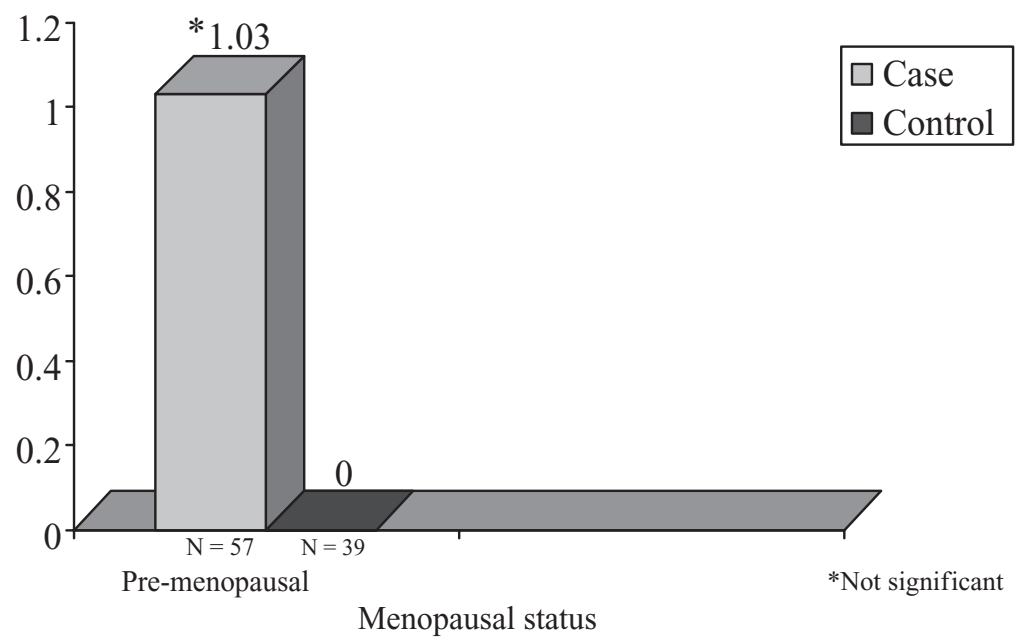

Figure I Median coronary calcium Agatson score in PCOS cases and controls by age $(<45)$ and menopausal status. 


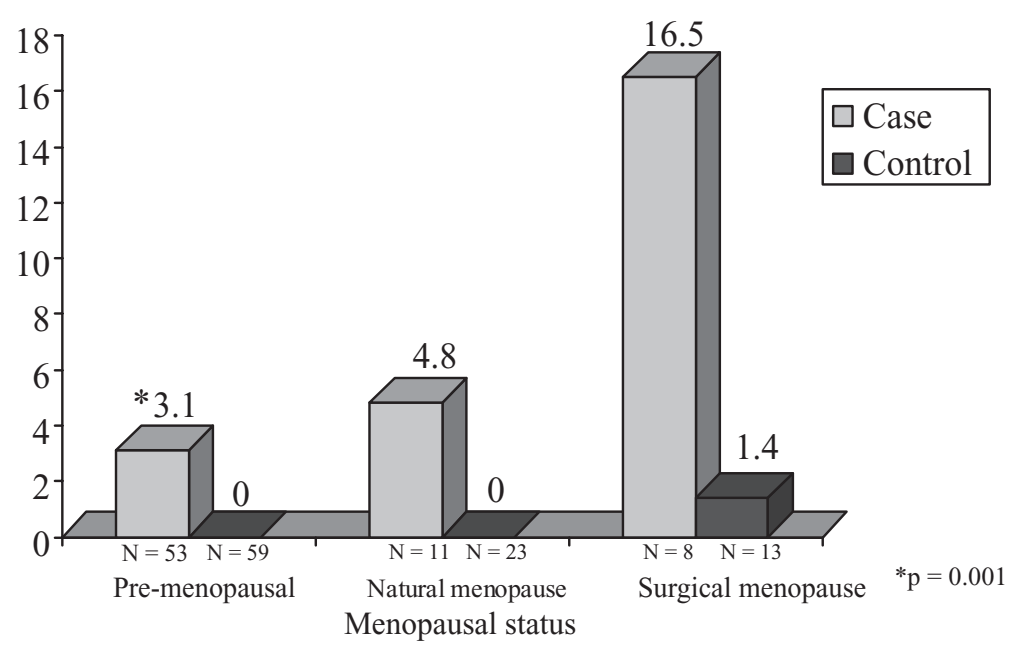

Figure 2 Median coronary calcium Agatson score in PCOS cases and controls by age (45-54) and menopausal status.

with PCOS case status $(\mathrm{p}=0.032)$, BMI as well as both surgical and natural menopause ( $\mathrm{p}=0.008$ and 0.037 respectively) in the overall case/control model. When considering PCOS cases separately (Table 5); BMI and surgical menopausal status with premenopausal status as the reference were significantly related to SCA (CAC values $\leq 10$ compared to $>10$ ) and within controls only (Table 6), natural menopause was significantly related to SCA as well as BMI. The parameter estimates (odds ratios) for both natural and surgical were both elevated when considering cases and controls separately; small numbers most likely affected the final $\mathrm{p}$ values.

\section{Discussion}

This study comprises one of the largest groups women with PCOS followed into middle age as they approach the menopause. The present study found that women with PCOS have an increased prevalence of CAC (63.1\%) compared to controls $(41.0 \%)$ after adjustment for age and BMI $(\mathrm{p}=0.037)$. The final model indicates that individuals (cases and controls) who have had a surgical/natural menopause have more sub clinical atherosclerosis independent of age and BMI and PCOS. PCOS remains a significant risk factor for $\mathrm{CAC}$ after adjustment for menopause, age, and BMI. When cases were considered separately, surgical menopause independent of age and BMI appeared to have the greatest effect as an independent risk factor for sub clinical atherosclerosis in PCOS cases; whereas among controls natural menopause was related to significantly increased CAC values. Both risk estimates (natural and surgical) were elevated in the separate models as well as in the combined dataset however. This stresses the importance of the menopausal transition, both natural and surgical in assessing cardiovascular risk.

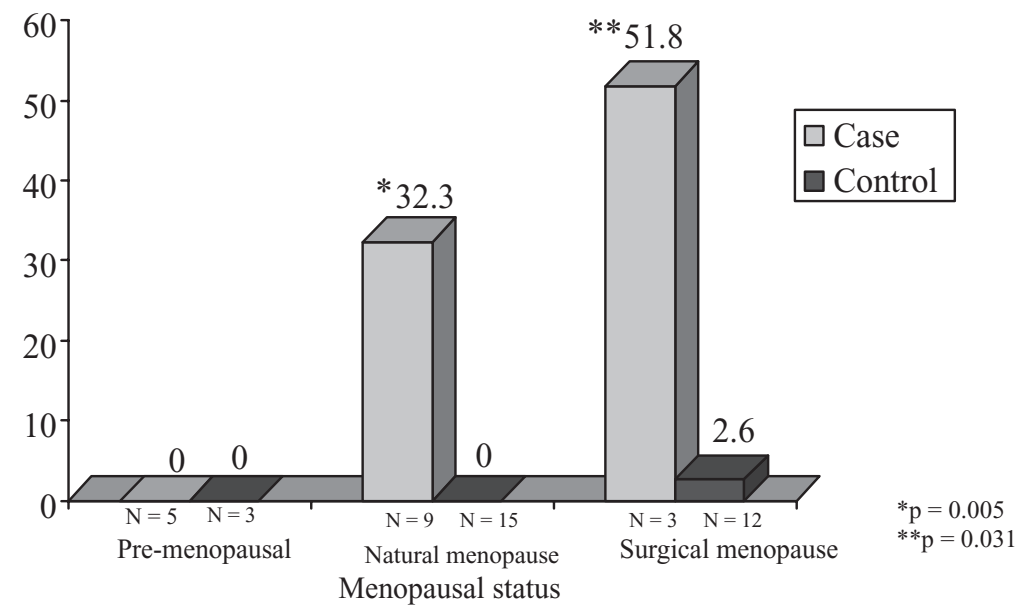

Figure 3 Median coronary calcium Agatson score in PCOS cases and controls by age (55+) and menopausal status. 
Table 4 Logistic regression models of CAC ( $\leq 10$ vs $10+)$ with CHD risk factors in PCOS cases and controls adjusted for age and $\mathrm{BMI}(\mathrm{N}=315)$

\begin{tabular}{|c|c|c|c|c|c|}
\hline Models & $\beta$ & SE & OR & $95 \% \mathrm{CI}$ for OR & pValue \\
\hline I.PCOS & 0.643 & 0.309 & 1.90 & $1.04-3.48$ & 0.037 \\
\hline 2.PCOS & 0.605 & 0.312 & 1.83 & $0.99-3.38$ & 0.053 \\
\hline HDLT & -0.018 & 0.011 & 0.98 & $0.96-1.00$ & 0.101 \\
\hline 3. PCOS & 0.614 & 0.315 & 1.85 & $\mathrm{I} .00-3.42$ & 0.051 \\
\hline Fasting glucose & 0.037 & 0.014 & 1.04 & $1.01-1.07$ & 0.009 \\
\hline 4. PCOS & 0.667 & 0.311 & 1.95 & $1.06-3.58$ & 0.032 \\
\hline \multicolumn{6}{|l|}{ Menopausal status } \\
\hline Premenopausal (baseline) & & & 1.00 & & \\
\hline Natural menopause & 0.490 & 0.457 & 3.70 & $1.4-9.6$ & 0.008 \\
\hline Surgical menopause & 0.550 & 0.509 & 3.13 & $1.1-9.14$ & 0.037 \\
\hline
\end{tabular}

African American PCOS cases were on average heavier and had greater median CAC scores than their White counterparts. African American PCOS women had greater CAC prevalence than African American controls. $(p<0.05)$. In multiple logistic regression analysis, ethnicity was not a significant predictor of CAC score independent of PCOS status, age, and BMI.

Fasting glucose was associated with increased CAC within cases and controls and lower HDLT was related to higher CAC scores within controls only. Moreover, Type 2 diabetes was reported by PCOS in $12.1 \%$ of cases and $1.8 \%$ of controls $(p<0.05)$. Calculated LDL and triglycerides were of borderline significance as risk factors of SCA in PCOS cases and controls.

In the baseline study (Talbott et al 1998) of this group of women comprised of 243 cases and 243 age matched controls, women with PCOS independent of age and BMI were found to have increases in cardiovascular risk factors in each ten year age strata beginning as early as 19 years of age. It was also noted in a second follow-up (1997-1999) of this population (Talbott et al 2000), evidence of increased average carotid (intima medial wall thickness) IMT among women with PCOS ( $45^{+}$years) compared to control subjects. This study involved 125 cases and 142 controls aged $30+$ in 1997-1999. Collection of baseline socio-demographic data, reproductive hormone levels and CHD risk factors had been measured 5 years earlier. Results indicated that overall and in the group aged 30-44 years, no difference was noted in mean carotid IMT between cases and controls; however women aged $>45$ years had greater IMT than controls $(0.78 \mathrm{~mm}$ vs $70 \mathrm{~mm})$ and this remained after adjustment for age and BMI. When additional risk factors were added in the regression model, fasting insulin and waist: hip ratio appeared to attenuate the relationship of PCOS and IMT suggesting that at least part of the observed associated may be driven

Table 5 Logistic regression models of CAC ( $\leq 10$ vs $10+)$ with CHD risk factors in PCOS cases adjusted for age and BMI $(\mathrm{N}=149)-($ PCOS cases only)

\begin{tabular}{|c|c|c|c|c|c|}
\hline Models & $\beta$ & SE & OR & $95 \% \mathrm{Cl}$ for $\mathrm{OR}$ & p Value \\
\hline I.Age & 0.198 & 0.058 & 1.22 & $1.09-1.36$ & 0.001 \\
\hline $\mathrm{BMI}$ & 0.377 & 0.063 & 1.46 & $1.29-1.65$ & $<0.001$ \\
\hline 2.Age & 0.201 & 0.058 & 1.22 & $1.09-1.37$ & 0.001 \\
\hline BMI & 0.394 & 0.070 & 1.48 & I.29-I.70 & $<0.001$ \\
\hline HDLT & 0.011 & 0.017 & 1.01 & $0.98-1.05$ & 0.524 \\
\hline 3. Age & 0.171 & 0.060 & 1.19 & $1.05-1.34$ & 0.004 \\
\hline BMI & 0.342 & 0.063 & 1.41 & $1.24-1.59$ & $<0.001$ \\
\hline Fasting glucose & 0.060 & 0.031 & 1.06 & $1.00-1.13$ & 0.057 \\
\hline 4. Age & 0.047 & 0.047 & 1.048 & $0.84-1.07$ & 0.310 \\
\hline BMI & 0.182 & 0.033 & 1.20 & $1.127-1.33$ & $<0.001$ \\
\hline \multicolumn{6}{|l|}{ Menopausal status } \\
\hline Premenopausal (baseline) & & & 1.00 & & \\
\hline Natural menopause & 0.973 & 0.666 & 2.65 & $0.72-9.8$ & 0.144 \\
\hline Surgical menopause & 2.26 & $0.81 \mathrm{I}$ & 9.64 & $1.96-47.2$ & 0.005 \\
\hline
\end{tabular}


Table 6 Logistic regression models of CAC ( $\leq 10$ vs 10+) with CHD risk factors in controls adjusted for age and BMI $(\mathrm{N}=166)$

\begin{tabular}{|c|c|c|c|c|c|}
\hline Model & $\beta$ & SE & OR & $95 \% \mathrm{Cl}$ for OR & p Value \\
\hline I.Age & 0.063 & 0.032 & 1.07 & $1.00-1.13$ & 0.048 \\
\hline BMI & 0.217 & 0.039 & 1.24 & I.15-I.34 & $<0.001$ \\
\hline 2.Age & 0.086 & 0.034 & 1.09 & $1.02-1.16$ & 0.012 \\
\hline BMI & 0.202 & 0.040 & 1.22 & $1.13-1.32$ & $<0.001$ \\
\hline HDLT & -0.033 & 0.015 & 0.97 & $0.94-1.00$ & 0.030 \\
\hline 3.Age & 0.065 & 0.032 & 1.07 & $1.00-1.14$ & 0.046 \\
\hline BMI & 0.210 & 0.040 & 1.23 & $1.14-1.33$ & $<0.001$ \\
\hline Fasting glucose & 0.025 & 0.016 & 1.02 & $0.99-1.06$ & $<0.134$ \\
\hline 4.Age & -0.052 & 0.062 & 0.949 & $0.84-1.07$ & 0.339 \\
\hline BMI & 0.205 & 0.044 & 1.227 & $1.13-1.34$ & $<0.001$ \\
\hline \multicolumn{6}{|l|}{ Menopausal status } \\
\hline Premenopausal (baseline) & & & 1.00 & & \\
\hline Natural menopause & 1.54 & 0.783 & 4.7 & $1.01-21.7$ & 0.049 \\
\hline Surgical menopause & 0.397 & 0.911 & 1.5 & $0.25-8.19$ & 0.662 \\
\hline
\end{tabular}

by central obesity and insulin resistance. Investigators have speculated that exposure at an early age to an adverse cardiovascular profile as observed among women with PCOS leads to premature atherosclerotic changes. In summary, recent work in various and methodologies appears to confirm our previous finding of increased SCA in women with PCOS in both the coronary and carotid vasculature.

This was the first investigation to consider the effect of subclinical atherosclerosis in women with PCOS and similar aged controls as they undergo the menopausal transition. PCOS continues to be an independent risk factor for SCA after adjustment for age, BMI and menopausal status. Age is extremely correlated with menopausal status; and when considered in a multiple regression most likely reduced the significance of the effect of "natural" menopause in cases. It is noteworthy that within the 45-54 and 55+ age strata the surgical menopause group of both cases and controls exhibited the most pronounced increased median CAC values. The possible interaction of menopause and $\mathrm{CAC}$ in women with PCOS warrants further investigation. The age at which cases and controls underwent surgical menopause was relatively young and is indicative of further loss of the coronary protection afforded normally menstruating women. Time since menopause was the greatest among the surgical menopause group giving greater credence to this finding. The scientific assumption has been that "the offending organ" the ovaries, which for a PCOS woman's lifetime produced the classic symptoms of hyperandrogenemia and metabolic derangement, would at menopause, be less likely to lead to additional cardiovascular and metabolic damage. The paradox of the present findings, ie, significant increased CAC levels in menopausal cases versus controls of similar age challenges this view. The implication is that the menopausal transition coupled with the lifelong increase in cardiovascular risk factors (obesity, hyperinsulinemia, increased LDL and decreased HDLT) continues to create an adverse environment and a process in women with PCOS that outpaces those of non-PCOS women.

A possible bias with regard to the PCOS group is the presence of chronic anovulation history in which no or very few periods are the norm rather than the rule. We determined that there was one PCOS case that was regularly anovulatory, ie, had few or no periods throughout her reproductive years. It will important to determine if these results are replicated in other populations of middle aged women with PCOS as well as in further follow up of this cohort as they transition through menopause.

\section{Disclosure}

Funding Support for this project: PCO NIH NHLBI Grant\#2 ROI HL44664.

\section{References}

Allain Cc, Poon LS, Chan CSG, et al. 1974. Enzymatic determination of total serum cholesterol. Clin Chem, 20:470-5.

Azziz R, Woods KS, Reyna R, et al. 2004. The prevalence and features of the polycystic ovary syndrome in an unselected population. J Clin Endocrinol Metabolism, 89:2745-9.

Bucolo G, David H. 1973. Quantitive determination of serum triglycerides by the use of enzymes. Clin Chem, 19:476-82.

Christian RC, Dumesic DA, Behrenbeck T, et al. 2003. Prevalence and predictors of coronary artery calcification in women with polycystic ovary syndrome. J Clin Endocrinol Metab, 88:2562-8.

Cole's Cross Reference Directory. 1993. Allegheny County and Greater Pittsburgh Area. 1991-1993. Lincoln, Neb: Cole Publications and Information Services. 
Dunaif A. 1995. Hyperandrogenic anovulation (PCOS): A unique disorder of insulin action associated with an increased risk of non-insulin-dependent diabetes mellitus. Am J Med, 98:33S-39S.

Ehrmann DA, Barnes RB, Rosenfield RL, et al. 1999. Prevalence of impaired glucose tolerance and diabetes in women with polycystic ovary syndrome. Diabetes Care, 22:141-6.

Friedewald WT, Levy RI, Fredrickson DS. 1972. Estimation of the concentration of low-density lipoprotein cholesterol in plasma, without use of the preparative ultracentrifuge. Clin Chem, 18:499-502.

Johnson BD, Merz CN, Braunstein GD. 2004. Determination of menopausal status in women: the NHLBI sponsored Women's Ischemia Syndrome (WISE) Study. Journal of Women's Health, 13:872-87.

Knochenhauer ES, Key TJ, Kahsar-Miller M, et al. 1998. Prevalence of the polycystic ovary syndrome in unselected black and white women of the southeastern United States: a prospective study. J Clinical Endocrino Metab, 83:3078-82.

Lakhani K, Hardiman P, Seifalian AM. 2004. Intima-media thickness of elastic and muscular arteries of young women with polycystic ovaries. Atherosclerosis, 175:353-9.

Matthews KA, Meilahn E, Kuller LH, et al. 1989. Menopause and risk factors for coronary heart disease. $N$ Engl J Med, 321:641-6.
Meyer C, McGrath BP, Cameron J, et al. 2005. Vascular dysfunction and metabolic parameters in polycystic ovary syndrome. J Clin Endocrin Metab, 90:4630-5.

Orio F Jr, Palomba S, Cascella T, et al. 2004. Early impairment of endothelial structure and function in young normal-weight women with polycystic ovary syndrome. J Clin Endocrin Metab, 89:4588-93.

Sutton-Tyrrell K, Lassila HC, Meilahn E, et al. 1998. Carotid atherosclerosis in premenopausal and postmenopausal women in its association with risk factors measured after menopause. Stroke, 29:1116-21.

Talbott E, Guzick D, Clerici A, et al. 1995. Coronary heart disease risk factors in women with polycystic ovary syndrome. Arterioscler Thromb Vasc Biol, 15:821-6.

Talbott E, Clerici A, Berga SL, et al. 1998. Adverse lipid and coronary heart disease risk profiles in young women with polycystic ovary syndrome: results of a case-control study. J Clin Epidemiol, 51:415-22.

Talbott EO, Zborowski JV, Rager JR, et al. 2004. Evidence for an association between metabolic cardiovascular syndrome and coronary and aortic calcification among women with polycystic ovary syndrome. $J$ Clin Endocrinol Metab, 89:5454-61.

Vural B, Caliskan E, Turkoz E, et al. 2004. Evaluation of metabolic syndrome frequency and premature carotid atherosclerosis in young women with polycystic ovary syndrome. Human Reproduction, 20:2409-13. 